\title{
Luminescent Studies of Fluorescent Chromophore-Doped Silica Aerogels for Flat Panel Display Applications
}

\author{
S.A.C. Glauser \\ H.W.H. Lee
}

This paper was prepared for submittal to the

Materials Research Society Meeting

San Francisco, $C A$

March 31-April 4, 1997

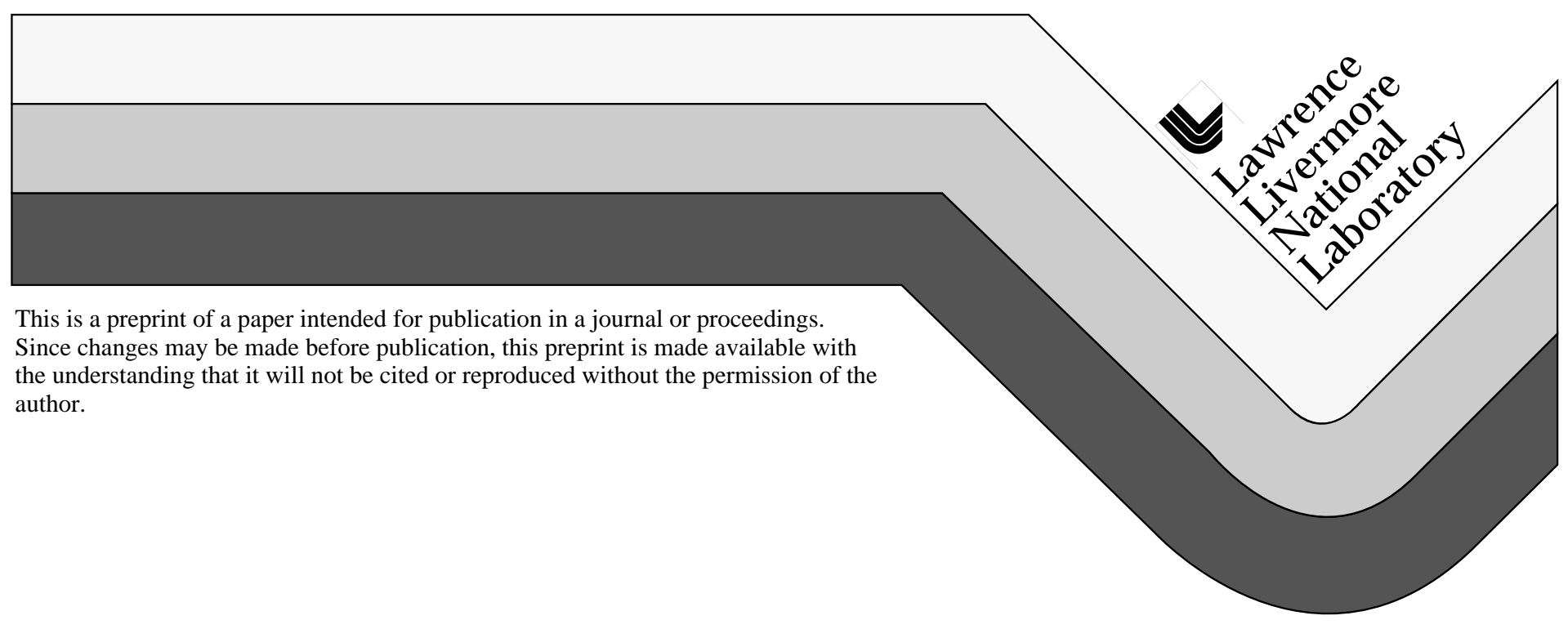




\section{DISCLAIMER}

This document was prepared as an account of work sponsored by an agency of the United States Government. Neither the United States Government nor the University of California nor any of their employees, makes any warranty, express or implied, or assumes any legal liability or responsibility for the accuracy, completeness, or usefulness of any information, apparatus, product, or process

disclosed, or represents that its use would not infringe privately owned rights. Reference herein to any specific commercial product, process, or service by trade name, trademark, manufacturer, or otherwise, does not necessarily constitute or imply its endorsement, recommendation, or favoring by the United States Government or the University of California. The views and opinions of authors expressed herein do not necessarily state or reflect those of the United States Government or the University of California, and shall not be used for advertising or product endorsement purposes. 


\section{LUMINESCENT STUDIES OF FLUORESCENT CHROMOPHORE-DOPED SILICA AEROGELS FOR FLAT PANEL DISPLAY APPLICATIONS}

SALLY A. C. GLAUSER 1 , HOWARD W. H. LEE 2

1. Department of Applied Science, University of California, Davis, CA 95616, sally@ wayfast.net

2. Lawrence Livermore National Laboratory, Livermore, CA 94551, hwhlee@llnl.gov

\section{ABSTRACT}

The remarkable optical and electronic properties of doped and undoped silica aerogels establish their utility as unique, multifunctional host materials for fluorescent dyes and other luminescent materials for display and imaging applications. We present results on the photoluminescence, absorption, and photoluminescence excitation spectra of undoped silica aerogels and aerogels doped with $\mathrm{Er}^{3+}$, rhodamine $6 \mathrm{G}(\mathrm{R} 6 \mathrm{G})$, and fluorescein.

\section{INTRODUCTION}

Aerogels represent a novel class of open-cell nanoporous materials with remarkable optical, electronic, thermal, and structural properties. Many of these properties are ideal for host matrices in which luminescent materials are incorporated for flat panel display (FPD) applications. Some of these properties include low weight, minimal optical and electronic interactions with the chromophore, and physical robustness. Silica aerogels can have densities as low as $3 \mathrm{mg} / \mathrm{cc}$, or roughly be $99.8 \%$ air and $0.2 \%$ material backbone. The backbone structure of colloidal aerogels is made up of clusters of materials which are 4 to $10 \mathrm{~nm}$ in diameter. They have interconnected pores and extensive internal surface areas ranging from 500 to $1200 \mathrm{~m}^{2} / \mathrm{g}$. Aerogels are physically robust, able to support 1600 times their own weight and stable in air and vacuum. Aerogels can be doped with a variety of atoms and molecules including metals, metal oxides, salts, and phosphors. In addition, silica aerogels are non-hazardous and non-flammable.

We studied the optical properties of silica aerogels doped with a luminescent materials

$\mathrm{Er}^{3+}$, rhodamine $6 \mathrm{G}$, and fluorescein. We report on the photoluminescence (PL), absorption, and photoluminescence excitation (PLE) spectra of theses doped aerogels, and evaluate their performance for FPD purposes. We contrast the optical properties of these doped aerogels with those observed for each chromophore and find that silica aerogels serve as an ideal host matrix. Our results demonstrate the potential utility of these silica aerogels as host materials for fluorescent dyes and other luminescent materials for display and imaging applications.

\section{EXPERIMENT}

The preparation of the aerogels studied in this paper is discussed in detail in reference 1 .

Transmission and absorption spectra were recorded with an absorption spectrophotometer. Optical excitation for PL studies was provided by three sources: (1) the 100 fs output from a self-modelocked Ti:sapphire laser was frequency-doubled with an appropriate KDP crystal to give excitation pulses varying from $355-400 \mathrm{~nm}$ and at a $82 \mathrm{MHz}$ repetition rate, (2) the $\sim 150 \mathrm{fs}$ output from a Ti:sapphire regenerative amplifier was frequency-doubled to give $400 \mathrm{~nm}$ excitation pulses at a $1 \mathrm{kHz}$ repetition rate, and (3) a commercial fluorimeter. Photoluminescence spectra were recorded with a fluorimeter and also with a $0.25 \mathrm{~m}$ monochromator equipped with an intensified optical multichannel analyzer. The PL spectra were corrected for the spectral response of the optical system. Photoluminescence excitation spectra were recorded with a fluorimeter.

\section{RESULTS}


Figure 1 shows the PL spectrum for a typical low density silica aerogel excited at $264 \mathrm{~nm}$. This spectrum can be compared with figures 2-4, which show the photoluminescence spectra for the chromophore-doped silica aerogels. The luminescence of the aerogel host is much smaller in magnitude than the intensity of the dopant in the aerogel. Also the peak of the luminescence of the undoped aerogel occurs far below the peaks of the doped aerogels.

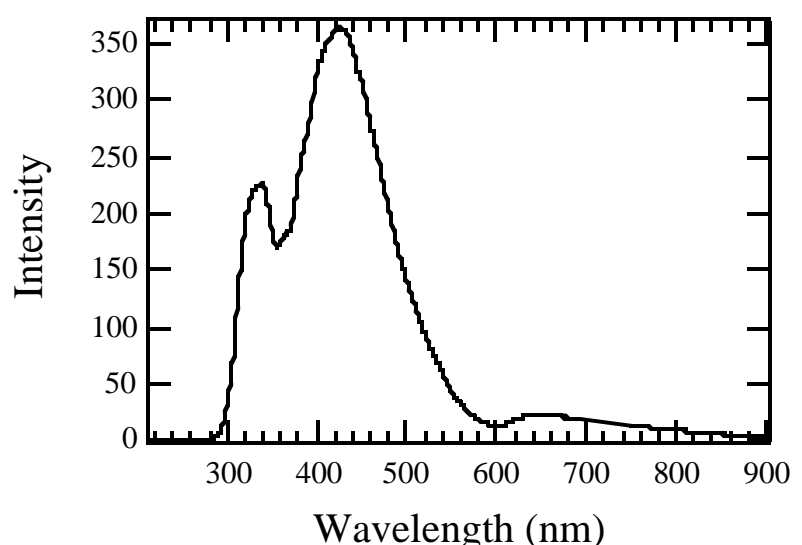

Figure 1. PL spectrum of a $30 \mathrm{mg} / \mathrm{cc}$ low density silica aerogel excited at $264 \mathrm{~nm}$.

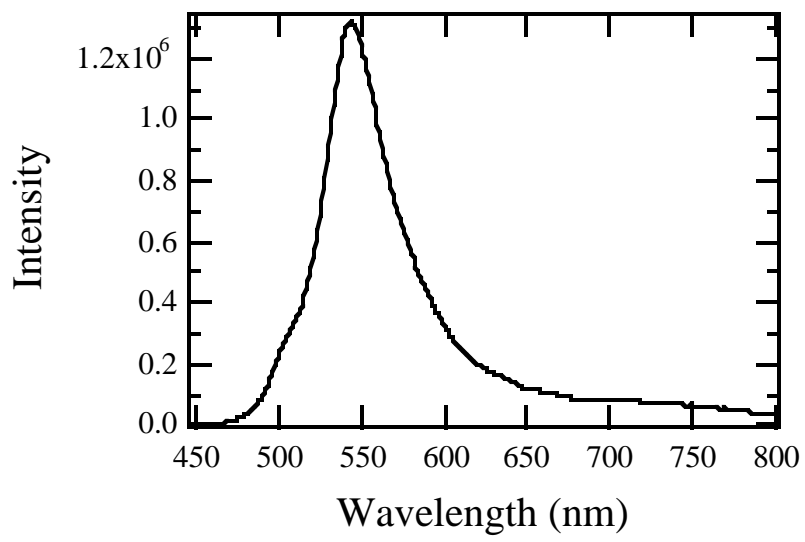

Figure 3. PL spectrum of Fluorescein in silica aerogel excited at $266 \mathrm{~nm}$.

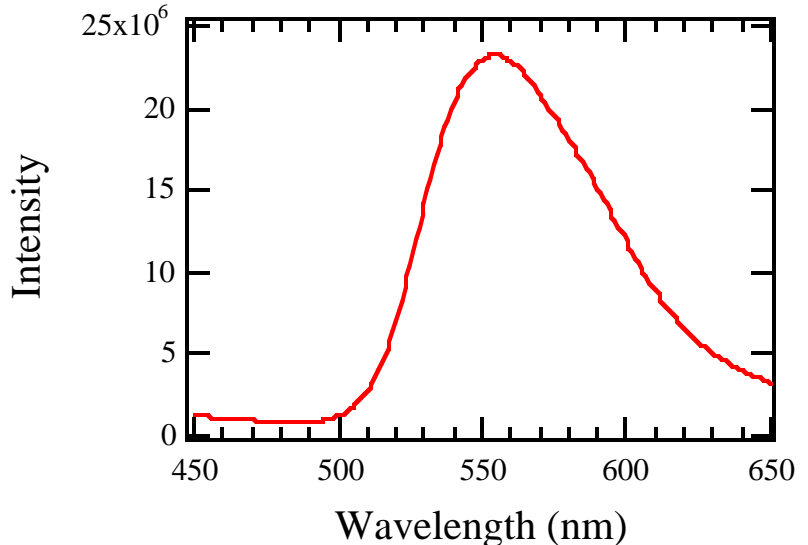

Figure 2. PL spectrum of R6G in silica aerogel excited at $365 \mathrm{~nm}$.

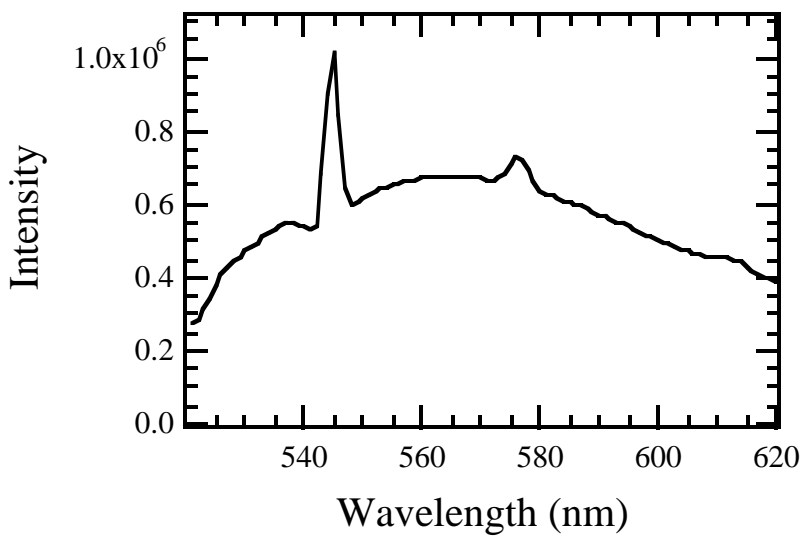

Figure 4. PL spectrum of $\mathrm{Er}^{3+}$ in silica aerogel excited at $450 \mathrm{~nm}$.

Absorption spectra are shown in figures 5-8 for undoped aerogel, R6G in aerogel, fluorescein in aerogel, and $\mathrm{Er}^{3+}$ in aerogel. Here again, we see that absorption in the aerogel does not affect the absorption of the doped aerogel. 

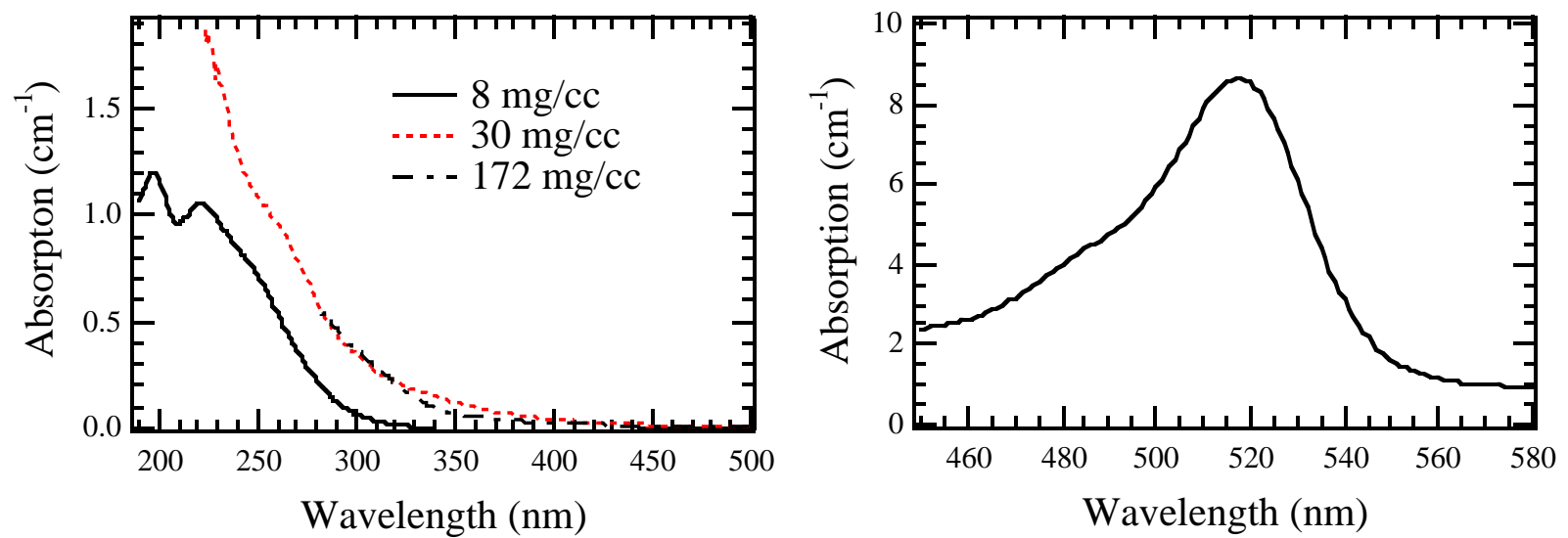

Figure 5. Absorption for different low Figure 6. Absorption peak for R6G in silica densities of silica aerogels.

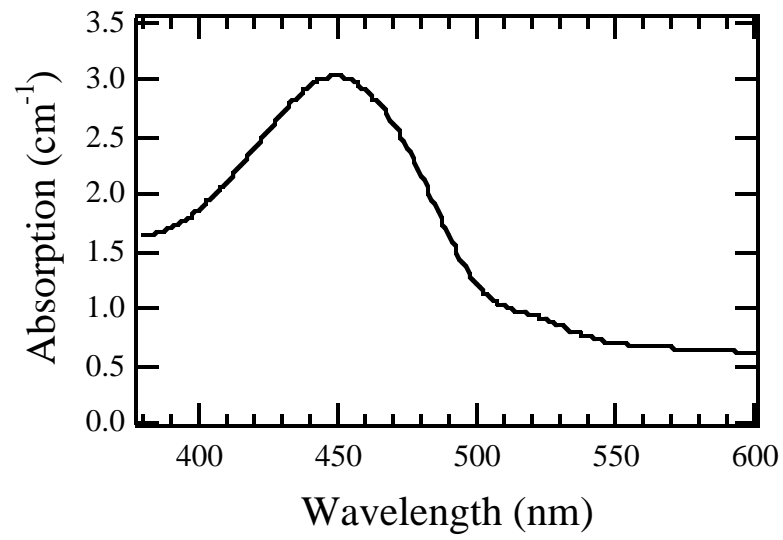

Figure 7. Absorption peak for Fluorescein in silica aerogel.

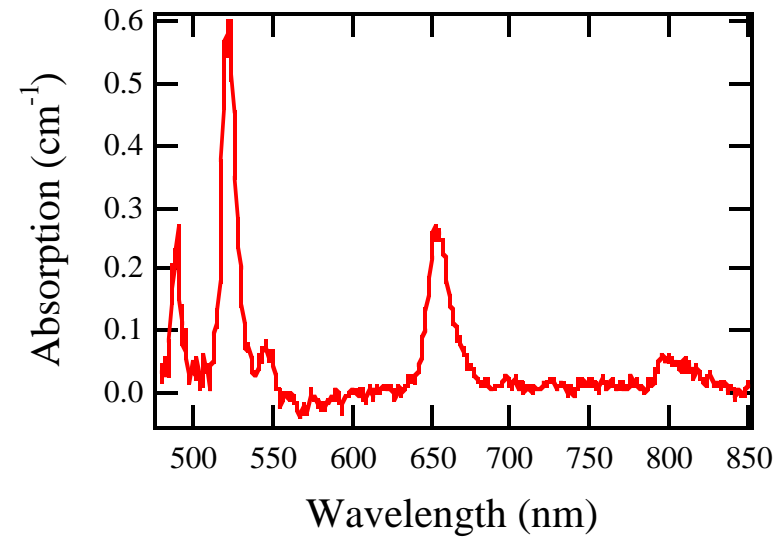

Figure 8. Absorption peaks for $\mathrm{Er}^{3+}$ in silica aerogel.

The PLE spectra are shown in figures 9-12 for undoped aerogel, R6G in aerogel, fluorescein in aerogel, and $\mathrm{Er}^{3+}$ in aerogel. In the PLE spectra we monitor the main PL peak in for each aerogel to ensure that the contribution to that peak is due solely to the dopant and not to the aerogel matrix.
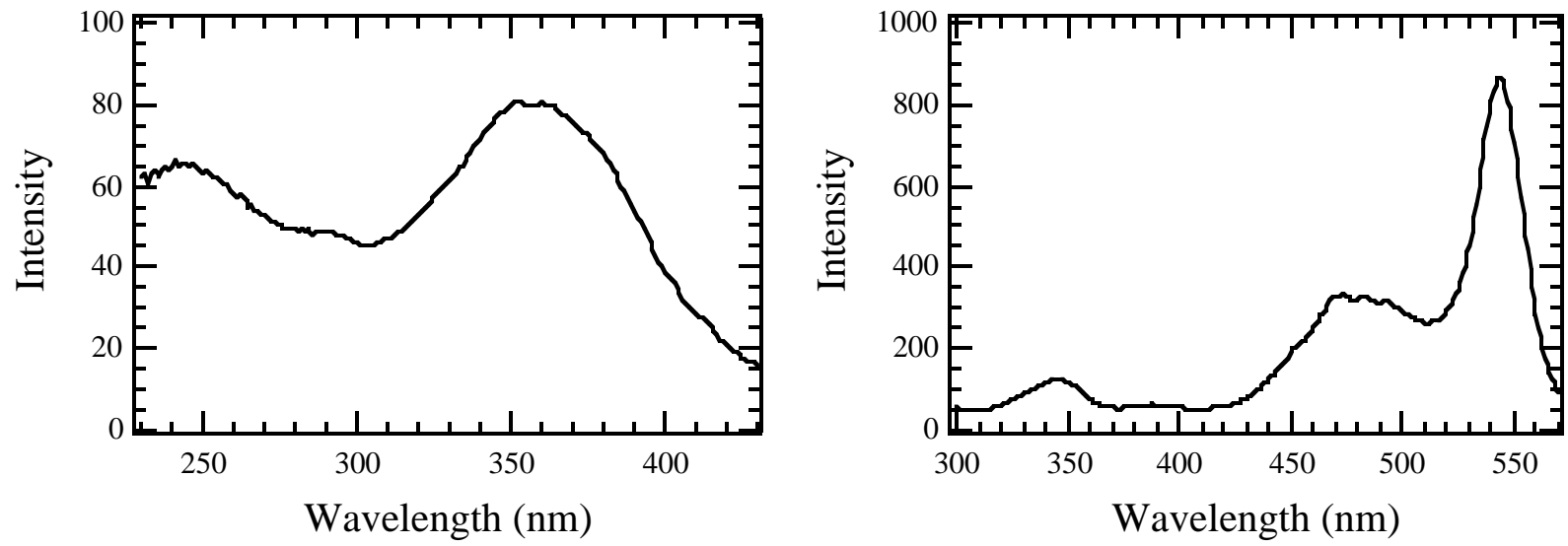

Figure 9. PLE spectrum of $30 \mathrm{mg} / \mathrm{cc}$ silica Figure 10. PLE spectrum of R6G in silica aerogel monitoring the PL peak at $428 \mathrm{~nm}$. aerogel monitoring the PL peak at $555 \mathrm{~nm}$. 


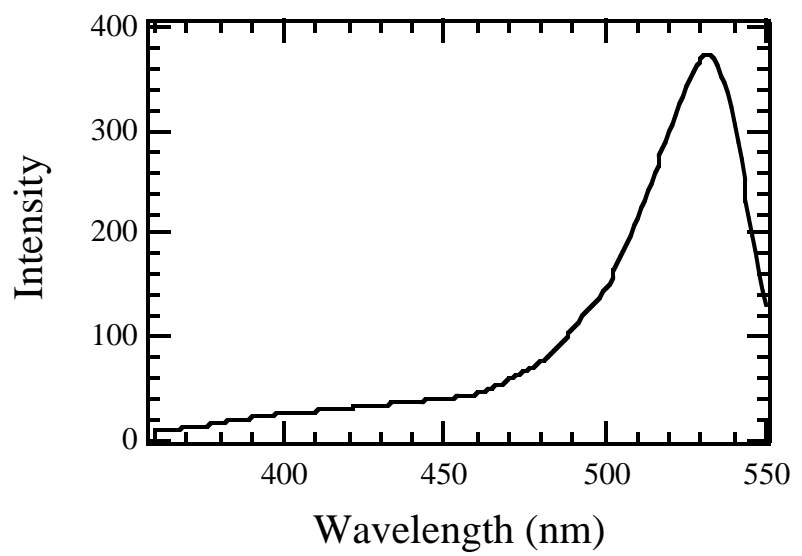

Figure 11. PLE spectrum of Fluorescein in silica aerogel monitoring the PL peak at 544 nm.

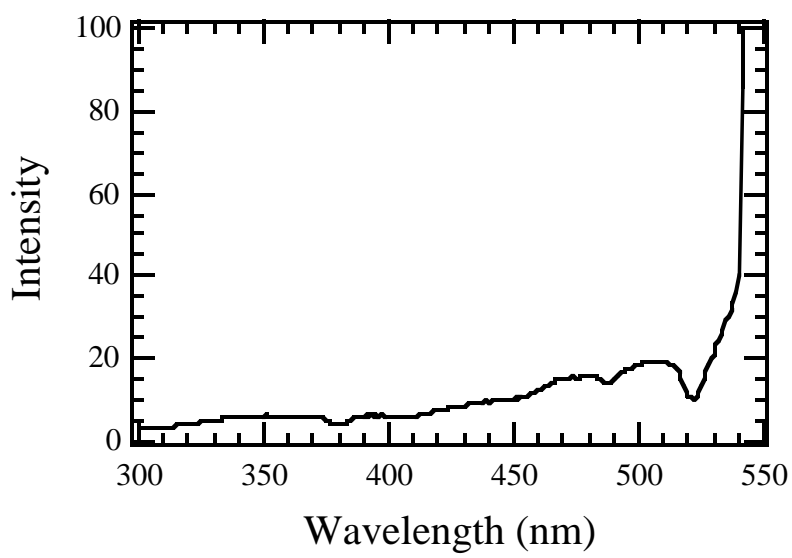

Figure 12. PLE spectrum of $\mathrm{Er}^{3+}$ in silica aerogel monitoring the PL peak at $545 \mathrm{~nm}$.

\section{CONCLUSIONS}

The PL peaks in aerogel occur at 318, 428, and $620 \mathrm{~nm}$. The absorption onset appears near $310 \mathrm{~nm}$. All peaks occur well below the bandedge of amorphous silica, indicating the presence of defects or surface states within the aerogel. For example, the non-stoichiometric sub-oxide $\mathrm{SiO}_{\mathrm{X}}$ has a smaller bandgap than $\mathrm{SiO}_{2}\left(\mathrm{E}_{\mathrm{g}} \geq 3-4 \mathrm{eV}\right.$ for $\left.\mathrm{x} \sim 1.4-1.6\right)$. The PLE spectra confirm that the contribution to the PL peaks of the doped aerogel are due to the dopant and not to the aerogel. The aerogel spectra are easily distinguishable from and do not interfere with the chromophore spectra.

In the R6G sample, we observed a PL peak at $555 \mathrm{~nm}$. This corresponds to a dimer transition in R6G with emission that is reported to be between $556-565 \mathrm{~nm}$.

Innocenzi, et. al. ${ }^{2}$ also show a PL shoulder at $515 \mathrm{~nm}$ that has not been assigned. Though we do not see a luminescence peak here, we do observe a strong absorption peak near $515 \mathrm{~nm}$. Our absorption spectrum also shows a shoulder at $490 \mathrm{~nm}$ that corresponds to the $500 \mathrm{~nm}$ dimer absorption of R6G.

Since the reported peak wavelength of the fluorescence of the fluorescein dimer is located at around 545 or $550 \mathrm{~nm}^{3}$, the PL peak in our spectrum, which is observed at $544 \mathrm{~nm}$, can reasonably be assigned to the dimer fluorescence. The absorption of fluorescein in methanol occurs at $498 \mathrm{~nm}$ which corresponds to our absorption peak.

In the $\mathrm{Er}^{3+}$ sample, we observe a strong $\mathrm{Er}^{3+} \mathrm{PL}$ peak at $545 \mathrm{~nm}$ and a weaker one at 578 $\mathrm{nm}$. Our absorption spectrum shows peaks at 488, 523, 545, 654, and $796 \mathrm{~nm}$. In comparison, $\mathrm{Xu}$, et. al. ${ }^{4}$ reported PL peaks from $\mathrm{Er}^{3+}$ doped sol-gel silica glass at 525, 550, and $660 \mathrm{~nm}$, which likely results from the absorption peaks we observed. The $550 \mathrm{~nm}$ PL is ascribed to a $4 \mathrm{~S}_{3 / 2}$ $\rightarrow 4 \mathrm{I}_{15 / 2}$ transition in $\mathrm{Er}^{3+}$.

In summary, the optical properties of silica aerogels make them ideal hosts for fluorescent dyes and other luminescent materials for electroluminescent applications. The photoluminescence, absorption, and photoluminescence excitation of chromophores such as rhodamine 6G, fluorescein, and $\mathrm{Er}^{3+}$ are not significantly altered when incorporated in an aerogel matrix. Their desirable luminescent properties (visible light emission, and high luminescent quantum efficiency) therefore should remain intact. Nearly any luminescent center can be used, allowing a wide variety in choice of dopant and therefore color. In addition, aerogels can be made into thin films or molded into any shape. Thus, aerogels are a unique, multifunctional host material for a variety of chromophores with applications in the display and imaging fields. 


\section{ACKNOWLEDGMENTS}

This work was performed under the auspices of the US Department of Energy by Lawrence Livermore National Laboratory under contract No.W-7405-ENG-48.

\section{REFERENCES}

1. T. M. Tillotson and L. W. Hrubesh, J. Non-Cryst. Solids, 145, p. 44 (1992).

2. P. Innocenzi, H. Kozuka, and T. Yoko, J. Non-Cryst. Solids, 210, p. 26 (1996).

3. T. Fujii, A. Ishii, and Y. Kurihara, Research on Chem. Intermediates, 19, p. 333 (1993).

4. W. Xu, S. Dai, L. M. Toth, G. D. Del Cul, and J. R. Peterson, J. Non-Cryst. Solids, 194, p. 235, (1996). 


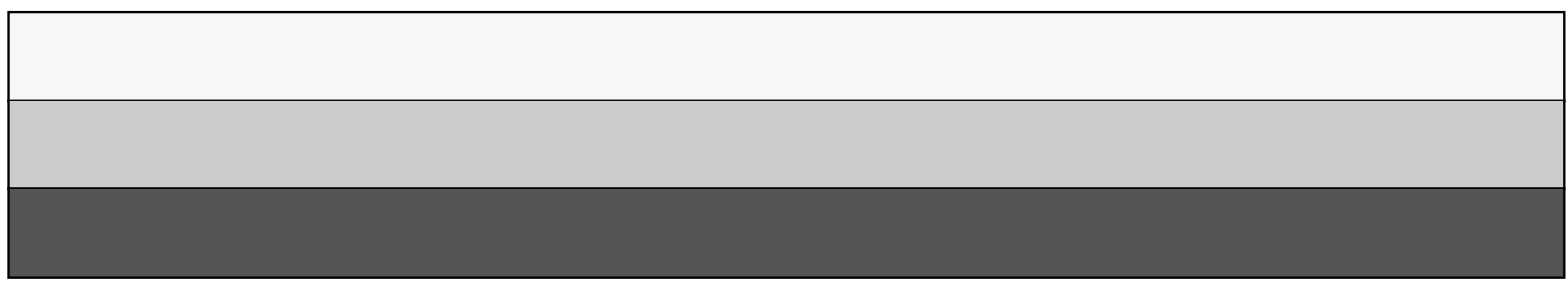

